\title{
Centralizers on a super-reflexive Schatten ideal
}

\author{
Jesús Suárez de la Fuente ${ }^{1}$
}

Received: 8 January 2018 / Accepted: 12 May 2018 / Published online: 29 May 2018

(C) Fondazione Annali di Matematica Pura ed Applicata and Springer-Verlag GmbH Germany, part of Springer Nature 2018

\begin{abstract}
We give a simple proof that there is no strictly singular bicentralizer on a superreflexive Schatten ideal. This result applies, in particular, to the $p$-Schatten class for $1<$ $p<\infty$.
\end{abstract}

Keywords Schatten $\cdot$ Strictly singular $\cdot$ Bicentralizer

Mathematics Subject Classification Primary: 46A16 - 46B07 · 46B20

\section{Main}

Let $H$ be a separable Hilbert space and denote by $\mathcal{B}(H)$ the algebra of all bounded linear operators on $H$. We will follow the deep work of Kalton [4], the interested reader may consider also [2,3], to introduce the following definitions. Let $E$ be a Köthe sequence space which is symmetric, then we define the corresponding Schatten ideal $\mathcal{C}_{E}$ to be the algebra of all operators $A: H \rightarrow H$ whose singular values $s_{n}(A)$ satisfy that $\left(s_{n}(A)\right) \in E$. We endow $\mathcal{C}_{E}$ with the norm $\|A\|_{E}=\left\|\left(s_{n}(A)\right)\right\|_{E}$. In this notation, the usual $p$-Schatten class is written as $C_{\ell_{p}}$. Let us recall that a map $\Omega: \mathcal{C}_{E} \rightarrow \mathcal{B}(H)$ is a bicentralizer provided for some constant $k=k(\Omega)$ we have

$$
\|\Omega(V A W)-V \Omega(A) W\|_{E} \leq k\|V\|\|A\|_{E}\|W\|,
$$

for $A \in \mathcal{C}_{E}$ and $V, W \in \mathcal{B}(H)$. We will also say that $\Omega$ is strictly singular if its restriction to every infinite-dimensional closed subspace is never bounded.

The author was supported by project MTM2016-76958-C2-1-P and project IB16056 from La Junta de Extremadura.

$凶$ Jesús Suárez de la Fuente jesus@unex.es

1 Departamento de Matemáticas, Avda. de Elvas s/n, Badajoz, Spain 
Regarding the $L_{p}$-spaces, the following remarkable result for centralizers was proved by Cabello Sánchez [1, Proposition 1].

Proposition 1 There is no strictly singular centralizer on $L_{p}$ for $0<p<\infty$.

The proof of the proposition above contains several simplifications that are of independent interest. However, the core of the argument is based on a result of Kalton contained in the aforementioned paper [4], namely that every centralizer on $L_{p}$ arises as a derivation from some interpolation process for $p>1$. The same work of Kalton contains a similar statement for the Schatten class [4, Theorem 8.3]. The aim of this short note is to observe that using this last result one may prove a claim equivalent to Proposition 1 but for the Schatten class $\mathcal{C}_{\ell_{p}}$, and even overstep the limits of the $p$-class.

Proposition 2 There is no strictly singular bicentralizer on a super-reflexive Schatten ideal.

Proof Let $\Omega$ denote a bicentralizer on $\mathcal{C}_{E}$. Then there exists Hermitian bicentralizers $\Omega_{1}, \Omega_{2}$ and a constant $K>0$ so that if $A \in \mathcal{C}_{E}$ then

$$
\left\|\Omega(A)-\Omega_{1}(A)-i \Omega_{2}(A)\right\|_{E} \leq K\|A\|_{E},
$$

see [4, Lemma 8.1]. Let us find a copy of $\ell_{2}$ where $\Omega_{1}$ and $\Omega_{2}$ are bounded and the same will follow, by (1), for $\Omega$. Thus, pick $\Omega_{1}$, our first Hermitian bicentralizer, and apply [4, Theorem 8.3] to find symmetric Köthe sequence spaces $E_{0}, E_{1}$ such that $\left(\mathcal{C}_{E_{0}}, \mathcal{C}_{E_{1}}\right)_{\frac{1}{2}}=\mathcal{C}_{\widetilde{E}}$ (where $\widetilde{E}$ and $E$ have equivalent norms) and $\Omega_{1}$ is equivalent to the bicentralizer induced by the previous interpolation formula, say $\widetilde{\Omega}_{1}$. We write this last claim in a formal language as there is some constant $K_{1}$ such that

$$
\left\|\Omega_{1}(A)-\widetilde{\Omega}_{1}(A)\right\|_{E} \leq K_{1}\|A\|_{E},
$$

for $A \in \mathcal{C}_{E}$. Now, let us give the copy of $\ell_{2}$ that makes the trick: Pick $\left(e_{n}\right)_{n=1}^{\infty}$ an orthonormal basis in $H$. For this basis, consider the span of the corresponding first column matrix, or in other words the natural embedding

$$
\begin{aligned}
& J: \ell_{2} \longrightarrow \mathcal{C}_{E} \\
& a=\left(a_{n}\right)_{n=1}^{\infty} \longrightarrow A=\sum_{n=1}^{\infty} a_{n}\left(\cdot \mid e_{1}\right) e_{n} .
\end{aligned}
$$

Writing

$$
A=\left(\sum_{n=1}^{\infty} a_{n} e_{n}\right)\left(\cdot \mid e_{1}\right)=\left\|\left(a_{n}\right)\right\|_{\ell_{2}}\left(\sum_{n=1}^{\infty} \frac{a_{n}}{\left\|\left(a_{n}\right)\right\|_{\ell_{2}}} e_{n}\right)\left(\cdot \mid e_{1}\right),
$$

one may easily deduce that $s_{1}(A)=\left\|\left(a_{n}\right)\right\|_{\ell_{2}}$ and $s_{n}(A)=0$ for $n \geq 2$. Therefore, the operator $J$ is well defined and the image spans a copy of $\ell_{2}$. Observe that $E$ does not play any special role here so that the same holds for $E_{0}$ and $E_{1}$. Indeed,

$$
\left\|\left(s_{1}(A), 0,0, \ldots\right)\right\|_{E}=\left\|\left(s_{1}(A), 0,0, \ldots\right)\right\|_{E_{0}}=\left\|\left(s_{1}(A), 0,0, \ldots\right)\right\|_{E_{1}}=s_{1}(A) .
$$

Thus, for a fixed $A=J(a)$, defining the constant function $F_{A}(z)=A$ where $z$ runs on the complex unit strip, the previous comments show that $F_{A}$ takes values in $\mathcal{C}_{E_{0}} \cap \mathcal{C}_{E_{1}}$. This gives that $F_{A}$ is a well-defined map in the space of functions of Calderón $\mathcal{F}\left(\mathcal{C}_{E_{0}}, \mathcal{C}_{E_{1}}\right)$ (see [5] for a clear exposition). And moreover, by (3),

$$
\left\|F_{A}\right\| \mathcal{F}\left(\mathcal{C}_{E_{0}}, \mathcal{C}_{E_{1}}\right)=\|A\|_{E} .
$$


Hence, $F_{A}$ is a $C$-bounded selection of $A$ for the quotient map $\delta_{1 / 2}: \mathcal{F}\left(\mathcal{C}_{E_{0}}, \mathcal{C}_{E_{1}}\right) \rightarrow C_{\widetilde{E}}$, where $C$ depends only on the equivalence constant between $E$ and $\widetilde{E}$. Since $F_{A}$ is constant, its derivative must be zero, that in particular means $\widetilde{\Omega}_{1}(A)=0$ (see [5, Page 1159] for a quick description of this phenomenon) and the same must hold for every $A \in J\left(\ell_{2}\right)$. Thus, by (2), one has that $\Omega_{1}$ is bounded when restricted to $J\left(\ell_{2}\right)$, that is

$$
\left\|\Omega_{1}(A)\right\|_{E} \leq K_{1}\|A\|_{E},
$$

for every $A \in J\left(\ell_{2}\right)$. The same arguments work to find a constant $K_{2}$ satisfying $\left\|\Omega_{2}(A)\right\|_{E} \leq$ $K_{2}\|A\|_{E}$ for every $A \in J\left(\ell_{2}\right)$. Using $K_{1}, K_{2}$ and the inequality (1), one may easily check that $\Omega$ is bounded on $J\left(\ell_{2}\right)$ which finishes the proof.

Corollary 1 There is no strictly singular bicentralizer on $C_{\ell_{p}}$ for $1<p<\infty$.

\section{References}

1. Cabello Sánchez, F.: There is no strictly singular centralizer on $L_{p}$. Proc. Am. Math. Soc. 142(3), 949-955 (2014)

2. Cwikel, M., Milman, M., Rochberg, R.: Nigel Kalton's work on differentials in complex interpolation. In: Kalton Selecta, N.J. (ed.) Contemporary Mathematicians, vol. 2, pp. 569-578. Birkhäuser/Springer, Berlin (2016)

3. Godefroy, G.: The Kalton calculus, Topics in functional and harmonic analysis, 57-68, Theta Ser. Adv. Math., 14, Theta, Bucharest (2013)

4. Kalton, N.J.: Differentials of complex interpolation processes for Köthe function spaces. Trans. Am. Math. Soc. 333(2), 479-529 (1992)

5. Kalton, N.J., Montgomery-Smith, S.: Interpolation of Banach Spaces. Handbook of the Geometry of Banach Spaces, vol. 2, pp. 1131-1175. North-Holland, Amsterdam (2003) 